\title{
Mechanical properties of DNA replication
}

\author{
Stuart A. Sevier \\ Center for Theoretical Biological Physics, Rice University, Houston, Texas 77005, USA \\ and Institute for Medical Engineering and Science, Massachusetts Institute of Technology, Cambridge, Massachusetts 02139, USA
}

(Received 10 July 2019; accepted 28 April 2020; published 3 June 2020)

\begin{abstract}
Central to the function of cellular life is the reading, storage, and replication of DNA. Due to the helical structure of DNA, a complicated topological braiding of new strands follows the duplication of the old strands. Even though this was discovered over 60 years ago, the nature of the physical barriers this presents have only recently been uncovered. In this article we construct a simple idealized model of DNA replication using only the most basic mathematical and mechanical elements needed. The resulting model assembles the relevant behaviors of DNA braiding, DNA supercoiling, topoisomerase action, and transcription into one framework. This may serve as a foundation for future work as well as to shed light on recently observed replication/replication and replication/transcription conflicts.
\end{abstract}

DOI: 10.1103/PhysRevResearch.2.023280

\section{INTRODUCTION}

For life to continue cells must create new versions of themselves. A central task in this process is the creation and proper segregation of a new copy of DNA. This new copy of DNA must be made while simultaneously leaving DNA available for important cellular functions such as transcription. Soon after the helical nature of DNA was uncovered $[1,2]$ it was realized that each parent strand serves as a template for two daughter strands [3] (deemed semiconservative replication). Consequentially, as the motor proteins responsible for opening and copying DNA track the helical groove of the parent strand, a combination of replicated strand braiding and unreplicated strand twisting occurs [4-7].

The ability for cells to undo this braided state, while undergoing normal functions, presents a central competition between DNA replication, topoisomerase action, and transcription which cells must maintain to successfully operate and divide. Consequentially, understanding the mathematical and physical nature of DNA replication is of the utmost importance in understanding many aspects of cellular function. Many biological aspects of DNA replication and function have been uncovered [8-10], however important aspects of DNA replication such as nonlocal effects and coordination in DNA damage, replication fork conflicts, and transcriptional interference [4,9,11-14] are unresolved.

Important recent experimental efforts have shed light on the mechanical nature of both braided naked [15] and chromosomal [16] DNA. However, no experiments have been done which capture the active real-time mechanical aspects of DNA replication as well as its interaction with important DNA based processes such as transcription. Consequen-

Published by the American Physical Society under the terms of the Creative Commons Attribution 4.0 International license. Further distribution of this work must maintain attribution to the author(s) and the published article's title, journal citation, and DOI. tially, a physical model that can simultaneously accommodate the relevant behaviors of DNA braiding, DNA supercoiling, topoisomerase action, and transcription into one theoretical framework is needed to understand the role of DNA mechanics in DNA replication.

In this article we construct a simple picture of the physical, mechanical process of DNA replication. We begin by dividing the system into replicated and unreplicated regions examining the role of DNA braiding and DNA supercoiling in each region respectively (see Fig. 1). We will not explicitly incorporate the mechanics of the replication machinery or differences between organisms but will identify the places where appropriate changes can be made to the model presented. We will instead focus on adaptable, general principles of the mathematical and physical nature of DNA replication. However, even within this simple approach, we can begin to understand the behavior of several important DNA replication related processes.

\section{MODEL}

The basic coordinates are the replication fork distance from the replication start site $x$, the angle of replicated strand braiding $\theta$, and the angle of over-twisting $\phi$ of the unreplicated region (Fig. 1). We will take $\theta$ and $\phi$ to be of opposite sign so that they are both positive quantities as a function of fork position. Due to the helical nature of DNA and the semiconservative nature of replication, these quantities must satisfy the below topological constraint during replication when no strand passages have occurred:

$$
\phi+\theta=x \omega_{0},
$$

where $\omega_{0}=1.85 \mathrm{~nm}^{-1}$ encodes the natural linking number of DNA.

As the replication fork progresses, the relative difficulty in twisting the unreplicated DNA or braiding the replicated strands determines the form of the functions $\phi(x), \theta(x)$. The remainder of this article will focus on the nature of these two functions in the context of the mechanical effects of DNA 
Idealized model of

DNA replication

replicated region

un-replicated region

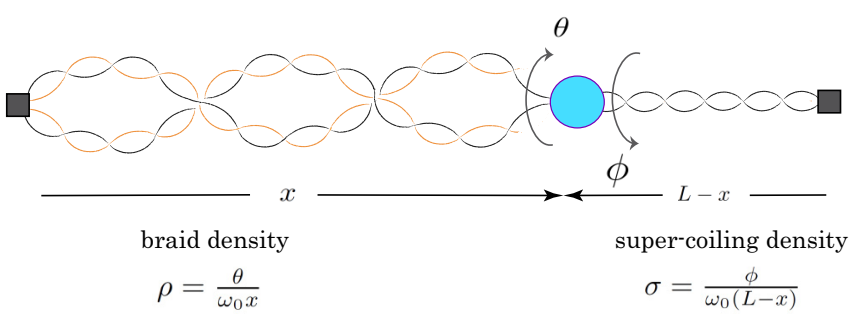

FIG. 1. A cartoon illustrating an idealized model of DNA replication. Fork progression shown at a distance $x$ from the start of replication results in braided replicated DNA strands rotated around each other $\theta$ times and supercoiled unreplicated DNA over-rotated $\phi$ times. The two regions are separated by idealized free rotating replication machinery shown in blue. The two ends are shown as simple topological barriers (gray squares) which prohibit the free rotation of the DNA thus conserving the linking number of the parental DNA. This results in a mechanical and topological connection between $\theta$ and $\phi$.

replication, topoisomerase action, and transcription. We will first study the case of an idealized topological barrier on either side which after studying will be adapted to a more realistic case where sites of transcription or additional DNA replication may take place.

To begin with, let us imagine a static barrier a distance $L$ from the start site of DNA replication (Fig. 1). This idealized barrier prevents free rotation of the unreplicated DNA as well as linking number conservation of the original parental DNA strands. A static barrier of this nature can be explicitly introduced in an in-vitro setting $[15,16]$ and may have natural or alternative forms in-vivo (discussed later). This boundary condition leads to a description of replicated DNA behavior through the replicated braid density (BD) $\rho(x)=\frac{\theta}{\omega_{0} x}$ and unreplicated supercoiling density (SCD) $\sigma(x)=\frac{\phi}{\omega_{0}(L-x)}$. Directly substituting the BD and SCDs into Eq. (1) yields a topological constraint on the densities

$$
(1-z) \sigma+z \rho=z,
$$

where the expression depends only on the fraction of the strand replicated $z=\frac{x}{L}$.

Without any means to anchor itself, the replication machinery cannot drive the two regions out of mechanical equilibrium thus making a clear torsional constraint between the replicated and unreplicated regions

$$
\tau_{\text {rep }}(\rho)=\tau_{\text {unrep }}(\sigma) .
$$

This constraint, combined with the topological constraints in Eq. (2) form the foundation for the most basic mechanical properties of DNA replication which will be examined in this article. These two observations must hold no matter the details of the replication machinery or detailed mechanical behavior of DNA on either side. Thus the formulation of the problem to this point is general in that either the mechanical response of naked or chromosomal DNA under various conditions are subject to the same constraints.
Generally, these expressions can be used as appropriate boundary conditions to solve transport equations (or in simulations) to obtain the full dynamics of the replicated and unreplicated regions, respectively. Here will only consider the case of the average, constant braid, and supercoiling density neglecting transport effects. We will adopt this perspective in our simple model due to DNA mechanical responses occurring on a subsecond timescale $[17,18]$ as well as the short distances of DNA expected between regions of replication and transcription [14]. In doing so we can then adopt the equilibrium torsional response of the two regions, resulting in mechanical responses due to constant braid and SC densities. Future work to address both of the issues will allow for insight into in-vivo experiments. However, the basic mechanical and topological constraints outlined above must still hold and here we choose to examine an abstract, adaptable first presentation of this process which may serve as a blueprint to construct more detailed, organism-specific models.

The determination of the BD $\rho$ and $\operatorname{SCD} \sigma$ is made through evaluating $\tau_{\text {rep }}(\rho)$ and $\tau_{\text {unrep }}(\sigma)$ during fork progression subject to the above constraints. To understand the mechanical competition between the replicated and unreplicated regions in its simplest form, we will explore the behavior of naked DNA under a constant force in conditions which are likely to occur in-vivo [19,20]. In this case the torsional response of the unreplicated region is given by an unbuckled elastic response followed by a buckled response with a constant torque [21]

$$
\tau_{\text {unrep }}(\sigma)= \begin{cases}\gamma \sigma, & \sigma<\sigma_{b}^{*}, \\ \tau_{b}, & \sigma_{b}^{*}<\sigma<\sigma_{c}^{*},\end{cases}
$$

where $\gamma, \tau_{b}$ are the elastic torsional coefficients and buckling torque, respectively, and $\sigma_{b}^{*}, \sigma_{c}^{*}$ are the SCDs at which buckling and a plectonemic collapse occur in the unreplicated region. The plectonemic collapse corresponds to the point at which the entire unreplicated region is expected to exist inside a plectoneme. The elastic response is on the order of $\gamma \approx$ $10^{2} \mathrm{pN} \mathrm{nm}$ and the force dependence described in [21]. Recent experimental results for the behavior of chromatin could easily be used instead [16] but will not be considered here.

In the replicated region the torsional response is due to the braiding of the two replicated double DNA strands. Since the common picture of DNA semiconservative replication involves freely rotating individual DNA strands, we will not consider the role of supercoiling in the replicated region. Additionally, because the torsion required to buckle braided DNA is higher than the constant torsion given after the unreplicated buckling transition $\tau_{b}$, we will not consider buckling in the braided, replicated strands. For the braided DNA to undergo buckling the unreplicated region must all be in a plectonemic state, resulting in a collapsed replication fork, a state which falls outside our framework of understanding DNA replication. Thus, we will only consider the torsional response associated with braiding two strands of naked DNA (due to the bending energy) [15]. Alternative formulations using the mechanical properties of braided chromosomal DNA could be made [16]. The resulting in nonlinear response to braid density is given by

$$
\tau_{\text {rep }}(\rho)=\alpha \rho^{3}
$$


with no force dependent quantities and $\alpha \approx 10^{4} \mathrm{pN} \mathrm{nm}$. The mechanical coefficients for both SC and braided DNA depend on temperature and physiological conditions [15,21] (see the Appendix for details) which will be fixed at the given values within this article.

Thus, using the torsional responses given by Eqs. (4) and (5) combined with Eq. (3) yields the constraint

$$
\alpha \rho^{3}= \begin{cases}\gamma \sigma, & \sigma<\sigma_{b}^{*}, \\ \tau_{b}, & \sigma_{b}^{*}<\sigma<\sigma_{c}^{*},\end{cases}
$$

which, through the use of the topological constraint on densities [Eq. (2)], can be used to obtain an equation explicitly for the braid density before buckling:

$$
\alpha \rho^{3}+\gamma \frac{z}{1-z} \rho=\gamma \frac{z}{1-z},
$$

while after buckling $\rho$ is given by the second line of Eq. (6). Since $\alpha \gg \gamma$ and $z<1$ we will drop the linear term (full solution given in the Appendix) to find the braid density as a function of replication position in the unbuckled and buckled regimes:

$$
\rho(z)= \begin{cases}\left(\frac{\gamma}{\alpha}\right)^{1 / 3}\left(\frac{z}{1-z}\right)^{1 / 3}, & z<z_{b}^{*}, \\ \rho_{b}, & z_{b}^{*}<z<z_{c}^{*},\end{cases}
$$

where the constant braid density $\rho_{b}=\left(\tau_{b} / \alpha\right)^{1 / 3}$ is given by the buckling torque in the unreplicated DNA and $z_{b}^{*}, z_{c}^{*}$ are the points of buckling and plectonemic collapse, respectively. Using Eq. (2) we can obtain the SCD in the unreplicated region

$$
\sigma(z)= \begin{cases}\frac{z}{1-z}\left[1-\left(\frac{\gamma}{\alpha}\right)^{1 / 3}\left(\frac{z}{1-z}\right)^{1 / 3}\right], & z<z_{b}^{*}, \\ \frac{z}{1-z}\left(1-\rho_{b}\right), & z_{b}^{*}<z<z_{c}^{*},\end{cases}
$$

which can be used to calculate the point at which both buckling and plectonemic collapse occur respectively as

$$
z_{b}^{*}=\frac{1}{1+\frac{\gamma}{\tau_{b}}}, \quad z_{c}^{*}=\frac{1}{1+\frac{1-\rho_{b}}{\sigma_{c}^{*}}},
$$

where the forms of $\tau_{b}$ and $\sigma_{c}^{*}$ are given in [21].

The torsional response as a function of DNA replication is given by Eq. (2) yielding

$$
\tau(z)= \begin{cases}\gamma \frac{z}{1-z}\left[1-\left(\frac{\alpha}{\gamma}\right)^{1 / 3}\left(\frac{z}{1-z}\right)^{1 / 3}\right], & z<z_{b}^{*}, \\ \tau_{b}, & z_{b}^{*}<z<z_{c}^{*} .\end{cases}
$$

So that for $x \ll L$ the braid density increases as $\rho(z) \sim$ $z^{1 / 3}, \sigma(z) \sim z, \tau(z) \sim z$ (see Fig. 2). As illustrated in Fig. 2, only a small fraction of DNA replication is possible before the replication fork enters a collapsed plectonemic state at $z_{c}^{*}$.

\section{TOPOISOMERASE ACTION}

To understand how DNA replication may avoid a plectonemic collapse or high torsion we must incorporate the action of topoisomerases, a class of molecular complexes capable of altering the linking number of DNA through strand breaks and passages [8]. In our simplified model this will be done through a dynamical equation for SCD which accounts for the generation of SCD during replication and its removal with

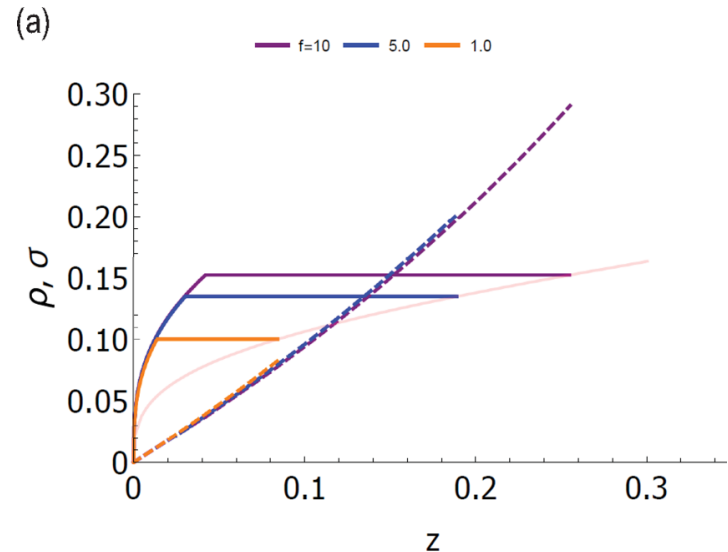

(b)

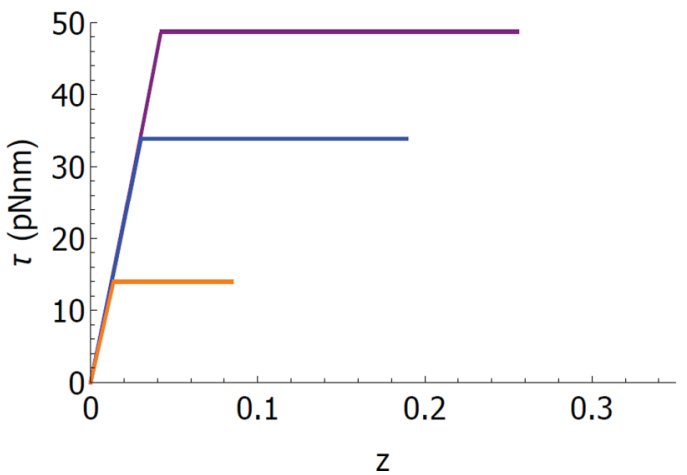

FIG. 2. Braid density $\rho$ (dashed), supercoiling density $\sigma$ (solid) (a) and torque $\tau$ (b) as a function of fork position $z=x / L$ at variance fixed forces (shown in top legend in $[\mathrm{pN}]$ ). Pink dashed curve shows the plectonemic collapse position $z_{c}$ for increased force. As the force in increased both the buckling $z_{b}$ and collapse $z_{c}$ increase but at the cost of increased buckling torque $\tau_{b}$.

topoisomerase. Using Eq. (2) we can generate the dynamical contribution of SCD due to replication fork progression

$$
(1-z) \dot{\sigma}-\dot{z} \sigma+z \dot{\rho}+\dot{z} \rho=\dot{z},
$$

which can be used to describe the dynamical state of the SCD in both the buckled and unbuckled state through the use of Eq. (6). This equation describes the dynamic response of the spatially homogeneous braid and SC densities and as before must hold no matter the details of the replication machinery or DNA on either side.

For the sake of simplicity and since we are interested in how topoisomerase action can prevent a replication collapse we will consider the case of constant $\rho$, given in the buckled phase, with a constant fork velocity $z(t)=v t / L$ to find

$$
\dot{\sigma}_{\text {rep }}=\frac{v}{L} \sigma\left(\frac{1}{z}+\frac{1}{1-z}\right)
$$

and will incorporate the action of topoisomerase with the addition of a a simple rate of removal $\dot{\sigma} \sim \lambda \sigma$. An additional term $\dot{\rho} \sim \kappa \rho_{b}$, as well as alternative terms for topoisomerase removal and SCD dependent velocity, can be included in this framework but will not be examined here. Additionally, we will not specify the direct mechanisms of topoisomerase action $[22,23]$ in an effort to identify the basic elements of this 


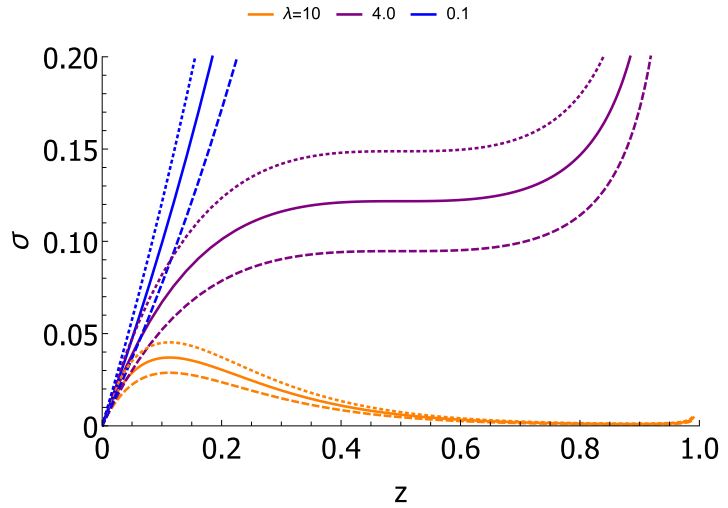

FIG. 3. Supercoiling density $\sigma$ as a function of fork position $z=x / L$ against an fixed barrier (solid) and a long gene convergently (dashed) or divergently (dotted) oriented for various topoisomerase action rates (top legend) at fixed force $f=1 \mathrm{pN}$. Depending on gene orientation SCD can be raised or lowered resulting in altered plectonemic collapse position. The $\frac{\tau_{b}}{D_{\mathrm{trx}}}$ contribution is dropped and $\frac{v_{\text {trx }}}{v}= \pm 1 / 5$ is used

dynamic competition. Recent experimental results have validated this approach, identifying the action of topoisomerase in the unreplicated region as the primary source of relaxation $[16,24]$. This yields a dynamical equation for the SCD

$$
\dot{\sigma}=\frac{v}{L} \sigma\left(\frac{1}{z}+\frac{1}{1-z}-\eta\right),
$$

where $\eta=\frac{\lambda L}{v}$ and we have used Eq. (2) to write the expression in its separable form. This equation can be directly integrated to find the solution

$$
\sigma(z)=\left(1-\rho_{b}\right) \frac{z}{1-z} e^{-\eta\left(z-z_{b}^{*}\right)},
$$

where we have used the boundary condition $\sigma\left(z_{b}^{*}\right)=\left(1-\rho_{b}\right)$ to account for matching at the buckling transition.

For a collapse to be avoided we must have $\sigma(z)<\sigma_{c}^{*}$ which puts a condition on the relative rates of replication $v$ and topoisomerase action $\lambda$ as well as the buckled density $\rho_{b}$. A local maxim can occur for $\sigma(z)$ at

$$
z^{*}=\frac{1}{2} \pm \sqrt{\frac{\eta-4}{4 \eta}}
$$

if the rates satisfy the inequality $\lambda>4 v / L$ it is possible for replication to occur over the entire system, as shown in Fig. 3. These results point to the importance of quick topoisomerase action during replication and offer insight into the importance of extra proteins used to stabilize plectonemes during replication [24]. Additionally, this effect should be directly observable using recent experimental setups [16] by changing the topoisomerase concentration and observing its effect on the amount of DNA replication possible.

\section{REPLICATION CONFLICTS}

During DNA replication additional topological barriers may exist to the free rotation of the unreplicated region presenting additional constraints for fork progression. A common obstruction to free DNA rotation is an active site of transcription. The interaction between fork progression and transcription can result in nonlocal interactions and stalling between transcription and replication [14]. We can incorporate the effect of an area of active transcription, and thus the absence of a static barrier, by changing the boundary condition at this point by introducing an additional degree of rotational freedom for DNA $\varphi$. Then the SCD in the unreplicated region (between the replication fork and the area of active transcription) is given by

$$
\sigma=\frac{\phi-\varphi}{\omega_{0}(L-x)}
$$

which leads to a modified Eq. (12):

$$
(1-z) \dot{\sigma}-\dot{z} \sigma+z \dot{\rho}+\dot{z} \rho+\frac{\dot{\varphi}}{\omega_{0} L}=\dot{z} .
$$

During transcription RNA polymerase can act as both a barrier to free DNA rotation as well as a source of SCD [25] leading to numerous interesting behaviors [26]. To incorporate this effect the dynamic mechanical response of $\varphi$ is given as

$$
\dot{\varphi}= \pm \omega_{o} v_{\mathrm{trx}}+\frac{\tau}{D_{\mathrm{trx}}},
$$

where $v_{\text {trx }}$ incorporates the ability of the gene to inject more SCD in the region which is positive (negative) when the gene is convergently (divergently) oriented with the direction of replication and $D_{\text {trx }}$ reflects the drag associated with difficulty in rotating the RNAP and nascent RNA (which may depend on the length of the gene).

The solution for Eq. (18) including nonlinear terms is difficult, however in the buckled case the nonlinear terms vanish and the action of topoisomerases can be included as before to find a modified Eq. (14):

$$
\dot{\sigma}_{\text {rep }}=\frac{v}{L} \sigma\left(\frac{1-\rho_{b} \mp \frac{v_{\text {trx }}}{v}-\frac{\tau_{b}}{D_{\text {trx }}}}{z}+\frac{1}{1-z}-\eta\right)
$$

yielding a solution

$$
\sigma(z)=\left(1-\rho_{b} \mp \frac{v_{\text {trx }}}{v}-\frac{\tau_{b}}{D_{\text {trx }}}\right) \frac{z}{1-z} e^{-\eta\left(z-z_{b}^{*}\right)} .
$$

As before this solution has a local maximum provided the rates satisfy Eq. (16). However the slope in Eq. (21) changes the effective buckling density, and thus the ability for $\sigma(z)<$ $\sigma_{c}^{*}$ during replication, as a function of gene length and orientation allowing for varying amounts of replication to occur before plectonemic collapse. This important difference is highlighted in Fig. 3 revealing the role of gene orientation in replication transcription conflicts. This effect could be responsible for additional phenomena related to transcriptional changes as a function of a gene's relative position and orientation to replication. Exploring these questions further may provide insight into the connection between the cell cycle and gene expression [27,28].

Other barriers could be incorporated in this framework resulting in modified topological and mechanical constraints. However, the central framework of this work should remain valid. For example, the presence of multiple replication forks can be accommodated resulting in modified equations for BD and SCDs as a function of total replication completed. 
Following the logic presented in the main text we can examine the behavior of multiple replication forks. Consider the case of two replication regions each of size $x_{1}, x_{2}$ of total length of $X$, convergently replicated into a region of $z$ for a total region of size $L=z+X$. To study this arrangement we must first notice that the same topological constraint must be true:

$$
\phi+\sum \theta_{i}=\omega_{0} X,
$$

where $\phi$ is the twist in the unreplicated between the replicated regions which have braided an amount $\theta_{i}$. This yields a generalized Eq. (2):

$$
z \sigma+x_{1} \rho_{1}+x_{2} \rho_{2}=\omega_{0} X
$$

where the SC and braid densities in each region are given by $\omega_{0} \sigma=\phi / z$ and $\omega_{0} \rho_{i}=\theta_{i} / x_{i}$, respectively.

Since the entire DNA is connected we will take the torsion in the entire region $L$ to be homogeneous. Thus we must have for each boundary between replicated and unreplicated regions $\tau_{\text {rep }}=\tau_{\text {unrep }}$ and thus we must have $\rho_{1}=\rho_{2}=\rho$ for all regions of unreplicated regions. Equation (23) then becomes

$$
\sigma=\frac{Z}{1-Z}(1-\rho)
$$

where $Z=X / L$ yielding a form which is identical to Eq. (2). Additional effects such as the role of topoisomerase could be added within this framework resulting in solutions closely following those presented for the case of one replication fork.

\section{CONCLUSION}

Many important aspects of DNA replication are not addressed here and the simple nature of the model developed in this article leaves a number of opportunities for future work to bridge the divide between theory and experiment. In this first effort we have only been concerned with developing a model that accommodates the equilibrium behavior of braided, replicated, and supercoiled, unreplicated DNA. In this vein we have neglected the behavior of the replication machinery during replication and the dynamic behavior of the braided and supercoiled DNA. Future models and experiments that incorporate the dynamical response of both regions may reveal important aspects of the process of DNA replication.

Even in the simple framework presented here, a number of important phenomena have been outlined, most notably the competition between topology and mechanics cells must overcome to successfully replicate DNA. During this process fork progression and the resulting torsional load put onto DNA must be dealt with before cells can divide and the basic elements of this competition have been highlighted in this article. Additionally, the basic properties of nonlocal interaction between replication and transcription have been outlined. This framework should serve as a foundation for future studies to bridge the divide between the simple properties of DNA replication and a more complete description needed to fully understand DNA replication in cells.

\section{ACKNOWLEDGMENTS}

This work was supported by the National Science Foundation Center for Theoretical Biological Physics (Grant NSF PHY-1427654). S.A.S. thanks Herbert Levine, Edward Banigan, Hugo Brandão, and Sumitabha Brahmachari for helpful discussions.

\section{APPENDIX}

\section{Phenomenological equations for braided DNA torsion}

We would like to find simple equations for the equilibrium torsional response of braided DNA as a function of braid density (BD). This can be obtained by analyzing the free energy density for braided DNA at equilibrium. Using [15] the free energy per unit length of a polymer with braid radius $R$ and pitch $P$ held at a fixed force $f$ is given by

$$
\beta \mathcal{E}_{b}=A \frac{R^{2}}{\left(R^{2}+P^{2}\right)^{2}}-\beta f \frac{P}{\sqrt{P^{2}+R^{2}}}+A^{-1} U(R, P),
$$

where $A=50 \mathrm{~nm}$ is the bending persistence of double stranded DNA with $\beta=1 / k_{b} T$ and $k_{b} T=4 \mathrm{pN} \mathrm{nm} \mathrm{(at}$ $290 \mathrm{~K})$ throughout this analysis.

The electrostatic energy $U(R, P)$ of the braid is given by

$$
U(R, P)=\xi K_{0}\left(\frac{2 R}{\lambda_{D}}\right)\left[1+0.828\left(\frac{R}{P}\right)^{2}+0.864\left(\frac{R}{P}\right)^{4}\right]
$$

where $\lambda_{D}$ is the Debye-screening length and $\xi$ is the amplitude of the Debye-Hückel potential, both of which depend on the chemical composition of the solution the braid is in [15]. We will use the electrostatic parameters corresponding to a salt concentration of $0.1 \mathrm{M}$.

To write the energy Eq. (A1) in terms of the braid density we can make the substitution $\rho=\theta /\left(\omega_{0} L\right)$ where $\theta$ is the angle by which the two strands of length $L$ have been braided and $\omega_{0}=2 \pi /(3.6 \mathrm{~nm})$ encodes the natural linking density of DNA. For a braid of fixed length $L$ we can use need the geometric relationship $L=\theta \sqrt{R^{2}+P^{2}}$ for the helical wrapping of the braid. This leads to the expression

$$
\omega_{0} \rho=\frac{1}{\sqrt{R^{2}+P^{2}}},
$$

where $\eta=2 \pi \omega_{o}$. Solving for the pitch yields

$$
P=\sqrt{\frac{1}{\left(\omega_{0} \rho\right)^{2}}-R^{2}}
$$

so that the energy per unit length is given by

$$
\beta \mathcal{E}_{b}=A R^{2}\left(\omega_{0} \rho\right)^{4}-\beta f P \omega_{0} \rho+A^{-1} U(R, \rho)
$$

substituting the density constraint from Eq. (A3) and expanding for small $\rho$ gives

$$
\beta \mathcal{E}_{b} \approx A R^{2}\left(\omega_{0} \rho\right)^{4}+\frac{1}{2} \beta f\left(\omega_{0} R \rho\right)^{2}-\beta f+A^{-1} U(R, \rho) .
$$

We can now minimize Eq. (A5) with respect to $R$ at fixed force and $\mathrm{BD} \rho$ yielding an effective energy density

$$
\mathcal{E}_{b}=\frac{\tilde{\alpha}}{4} \rho^{4}+f \frac{\tilde{\kappa}}{2} \rho^{2}-f,
$$




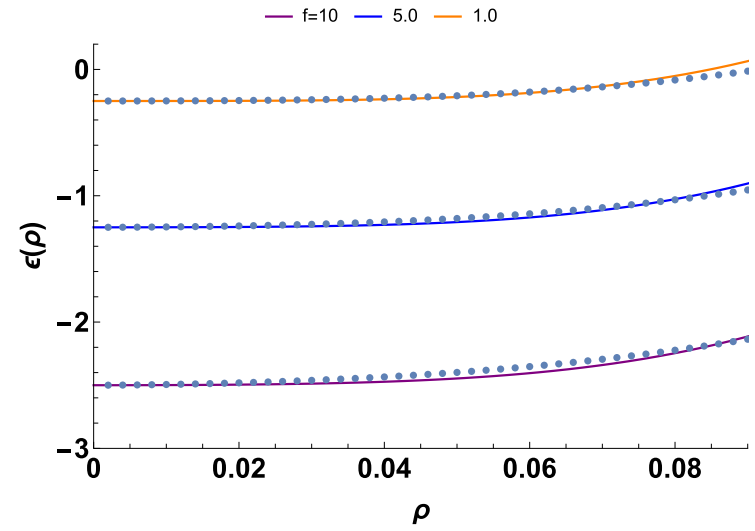

FIG. 4. Comparison of the free energy given by phenomenological Eq. (A8) to minimization of the full energy in Eq. (A5) for a $0.1 \mathrm{M}$ solution.

where $\tilde{\alpha}=k_{b} T 4 A R^{* 2} \omega_{0}^{4}$ and $\tilde{\kappa}=\omega_{0}^{2} R^{* 2}$. The resulting radius $R^{*}$ after minimization has little $\rho$ dependence and we have thus dropped the electrostatic contribution in the effective energy density $\mathcal{B}$. This is done as when minimized it contributes little to the energy density serving mainly to set the braid radius $R^{*}$. This radius is set by the electrostatic contribution by the energy which is driven by short distance electrostatic interactions. Comparisons between the full minimization of Eq. (A1) to the phenomenological equation are shown in Fig. 4.

Finally, the torsional response of braided DNA is given by the relationship

$$
\tau=\frac{1}{\omega_{0}} \partial_{\rho} \mathcal{B}=\alpha \rho^{3}+f \kappa \rho,
$$

where for physiological conditions stated above the resulting values for the phenomenological equations are $\alpha=\tilde{\alpha} / \omega_{0} \approx$ $13781 \mathrm{pN} \mathrm{nm}$ and $\kappa=\tilde{\kappa} / \omega_{0} \approx 7.7 \mathrm{~nm}$. Since $z<=1$ and $\alpha \gg \kappa$ and we can the linear term in the torsional analysis presented in the main text. A full solution to the SCD as a function of fork position including the linear $\rho$ terms is given below.

\section{Comparison to full solution}

It is possible to obtain a solution for the BD during replication with linear $\rho$ which were dropped in the analysis presented within the article. To do this let us follow the main text but now using

$$
\tau_{\text {rep }}(\rho)=\alpha \rho^{3}+f \kappa \rho
$$

for the torsional response of the replicated region we find the below equation for the $\mathrm{BD}$ as a function of fork position before buckling

$$
\alpha \rho^{3}+\left(f \kappa+\gamma \frac{x}{L-x}\right) \rho=\gamma \frac{x}{L-x}
$$

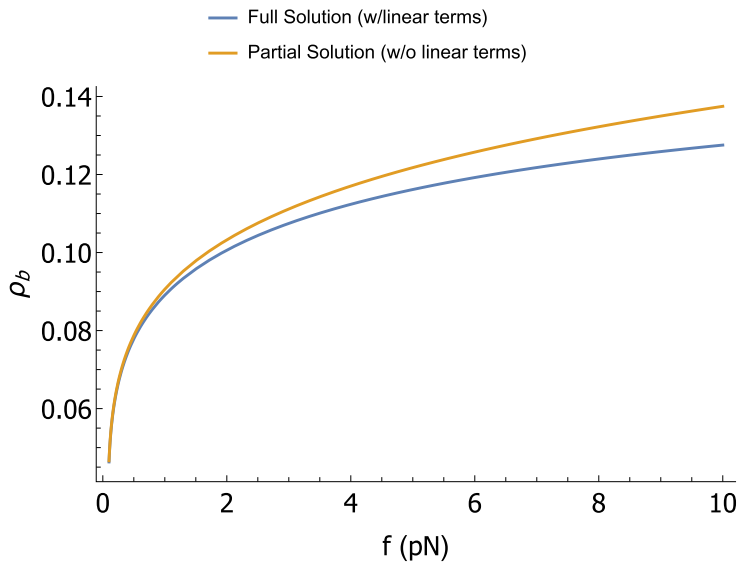

FIG. 5. Buckled $\rho_{b}$ comparison between the full solution given in the Appendix and partial solution used in the main text.

and after buckling

$$
\alpha \rho^{3}+f \kappa \rho=\tau_{b} .
$$

As discussed in the main text $\tau_{b}$ sets the torque in the system given by the buckled response of the unreplicated region. These equations yield a nonlinear solution for the braid density as a function of replication position

$$
\rho(x)= \begin{cases}\frac{1}{2^{1 / 33^{2 / 3} \alpha}} \Delta^{1 / 3}-\left(\frac{2}{3}\right)^{1 / 3}\left(\gamma \frac{x}{L-x}\right) \Delta^{-1 / 3}, & \rho<\rho_{b}, \\ \rho_{b}, & \rho_{b}<\rho,\end{cases}
$$

with

$$
\begin{aligned}
\Delta= & 9 \alpha^{2} \gamma \frac{x}{L-x} \\
& +\sqrt{4 \alpha^{3}\left(f \kappa+\gamma \frac{x}{L-x}\right)^{3}+27 \alpha^{4}\left(\gamma \frac{x}{L-x}\right)^{2}}
\end{aligned}
$$

and

$$
\rho_{b}=\frac{Q^{1 / 3}}{2^{1 / 3} 3^{2 / 3} \alpha}-\frac{f \kappa(2 / 3)^{1 / 3}}{Q^{1 / 3}}
$$

with

$$
Q=9 \alpha^{2} \tau_{b}+\sqrt{12 \alpha^{3}(\kappa f)^{3}+3^{4} \alpha^{4} \tau_{b}^{2}},
$$

which is compared to the simplified expression for $\rho_{b}$ used in the text in Fig. 5. Having obtained the BD, the solution for the SCD using the full solution follows in the same manner outlined in the main text.
[1] J. D. Watson and F. H. C. Crick, Nature (London) 171, 964 (1953).
[2] M. H. F. Wilkins, A. R. Stokes, and H. R. Wilson, Nature (London) 171, 738 (1953). 
[3] M. Meselson and F. W. Stahl, Proc. Natl. Acad. Sci. 44, 671 (1958).

[4] L. Postow, N. J. Crisona, B. J. Peter, C. D. Hardy, and N. R. Cozzarelli, Proc. Natl. Acad. Sci. USA 98, 8219 (2001).

[5] S. A. Schalbetter, S. Mansoubi, A. L. Chambers, J. A. Downs, and J. Baxter, Proc. Natl. Acad. Sci. USA 112, E4565 (2015).

[6] D. P. Bloch, Proc. Natl. Acad. Sci. USA 41, 1058 (1955).

[7] J. C. Wang, Untangling the Double Helix DNA Entanglement and the Action of the DNA Topoisomerases (2009).

[8] D. Racko, F. Benedetti, J. Dorier, and A. Stasiak, Nucleic Acids Res. 47, 521 (2019).

[9] S. Lambert and A. M. Carr, Chromosoma 122, 33 (2013).

[10] Y. Pommier, Y. Sun, S.-y. N. Huang, and J. L. Nitiss, Nat. Rev. Mol. Cell Biol. 17, 703 (2016).

[11] T. Kelly and A. J. Callegari, Proc. Natl. Acad. Sci. USA 116, 4973 (2019).

[12] J. E. Graham, K. J. Marians, and S. C. Kowalczykowski, Cell 169, 1201 (2017).

[13] T. R. Beattie, N. Kapadia, E. Nicolas, S. Uphoff, A. J. Wollman, M. C. Leake, and R. Reyes-Lamothe, eLife 6, 21763 (2017).

[14] K. S. Lang and H. Merrikh, Annu. Rev. Microbiol. 72, 71 (2018).

[15] S. Brahmachari and J. F. Marko, Phys. Rev. E 95, 052401 (2017).
[16] T. T. Le, X. Gao, S. h. Park, J. Lee, J. T. Inman, J. H. Lee, J. L. Killian, R. P. Badman, J. M. Berger, and M. D. Wang, Cell 179, 619 (2019).

[17] A. Crut, D. A. Koster, R. Seidel, C. H. Wiggins, and N. H. Dekker, Proc. Natl. Acad. Sci. USA 104, 11957 (2007).

[18] M. van Loenhout, M. de Grunt, and C. Dekker, Science 338, 94 (2012).

[19] G. Charvin, J.-F. Allemand, T. Strick, D. Bensimon, and V. Croquette, Contemp. Phys. 45, 383 (2004).

[20] J. F. Marko, Physica A 418, 126 (2015).

[21] J. F. Marko, Phys. Rev. E 76, 021926 (2007).

[22] M. Nöllmann, M. D. Stone, Z. Bryant, J. Gore, N. J. Crisona, S. Hong, S. Mitelheiser, A. Maxwell, C. Bustamante, and N. R. Cozzarelli, Nat. Struct. Mol. Biol. 14, 264 (2007).

[23] R. E. Ashley, A. Dittmore, S. A. McPherson, C. L. Turnbough, K. C. Neuman, and N. Osheroff, Nucleic Acids Res. 45, 9611 (2017).

[24] M. Guo, D. Haakonsen, W. Zeng, and S. M. Cell, Cell 175, 583 (2018).

[25] S. A. Sevier and H. Levine, Phys. Rev. Lett. 118, 268101 (2017).

[26] S. A. Sevier and H. Levine, Nucleic Acids Res. 46, 5924 (2018).

[27] V. Vijayan, R. Zuzow, and E. K. O'Shea, Proc. Natl. Acad. Sci. USA 106, 22564 (2009).

[28] M. Wang, J. Zhang, H. Xu, and I. Golding, Nat. Microbiol. 4, 2118 (2019). 